\title{
Article
}

\section{Ultrafast Deactivation of an Excited Cytosine-Guanine Base Pair in DNA}

Gerrit Groenhof, Lars V. Schfer, Martial Boggio-Pasqua, Maik Goette, Helmut Grubmller, and Michael A. Robb J. Am. Chem. Soc., 2007, 129 (21), 6812-6819• DOI: 10.1021/ja069176c • Publication Date (Web): 08 May 2007

\section{Downloaded from http://pubs.acs.org on March 23, 2009}

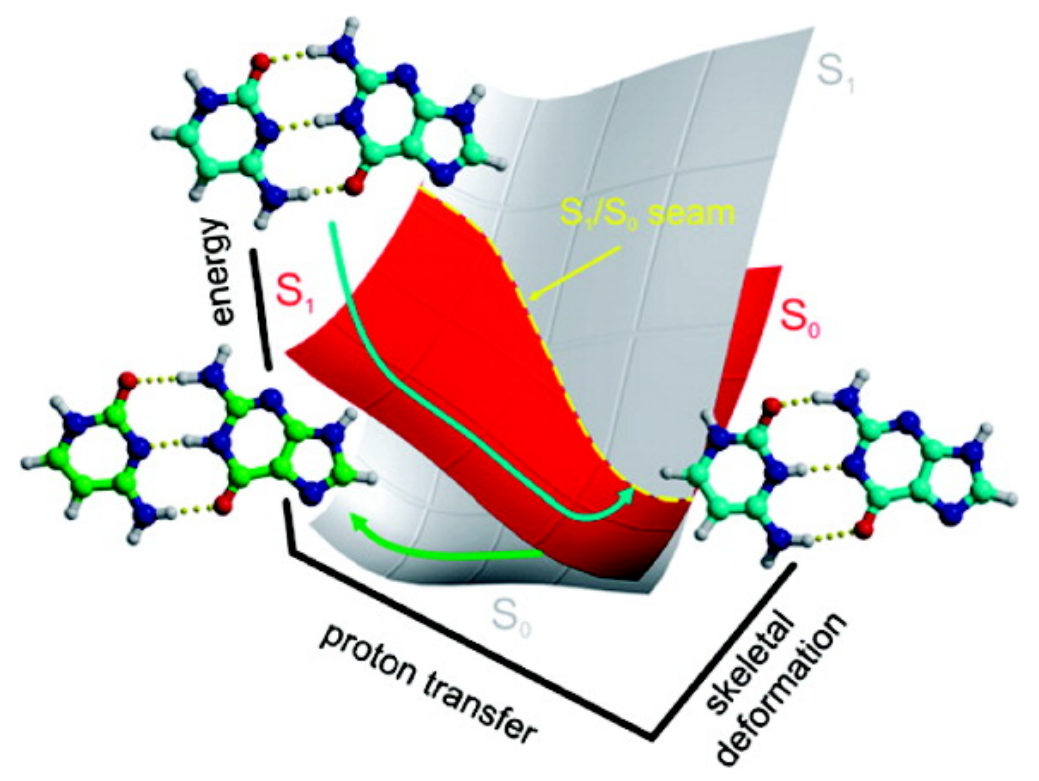

\section{More About This Article}

Additional resources and features associated with this article are available within the HTML version:

- $\quad$ Supporting Information

- $\quad$ Links to the 7 articles that cite this article, as of the time of this article download

- Access to high resolution figures

- $\quad$ Links to articles and content related to this article

- Copyright permission to reproduce figures and/or text from this article

\section{View the Full Text HTML}

\section{ACS Publications}




\title{
Ultrafast Deactivation of an Excited Cytosine-Guanine Base Pair in DNA
}

\author{
Gerrit Groenhof, ${ }^{\dagger}$ Lars V. Schäfer, ${ }^{\dagger}$ Martial Boggio-Pasqua, ${ }^{\ddagger}$ Maik Goette,${ }^{\dagger}$ \\ Helmut Grubmüller, ${ }^{\dagger}$ and Michael A. Robb*,‡ \\ Contribution from the Department of Theoretical and Computational Biophysics, \\ Max-Planck-Institute for Biophysical Chemistry, Am Fassberg 11, D-37077 Göttingen, Germany, \\ and Department of Chemistry, Imperial College, London SW 2AZ, U.K.
}

Received December 31, 2006; E-mail: mike.robb@imperial.ac.uk

\begin{abstract}
Multiconfigurational ab initio calculations and QM/MM molecular dynamics simulations of a photoexcited cytosine-guanine base pair in both gas phase and embedded in the DNA provide detailed structural and dynamical insights into the ultrafast radiationless deactivation mechanism. Photon absorption promotes transfer of a proton from the guanine to the cytosine. This proton transfer is followed by an efficient radiationless decay of the excited state via an extended conical intersection seam. The optimization of the conical intersection revealed that it has an unusual topology, in that there is only one degeneracylifting coordinate. This is the central mechanistic feature for the decay both in vacuo and in the DNA. Radiationless decay occurs along an extended hyperline nearly parallel to the proton-transfer coordinate, indicating the proton transfer itself is not directly responsible for the deactivation. The seam is displaced from the minimum energy proton-transfer path along a skeletal deformation of the bases. Decay can thus occur anywhere along the single proton-transfer coordinate, accounting for the remarkably short excitedstate lifetime of the Watson-Crick base pair. In vacuo, decay occurs after a complete proton transfer, whereas in DNA, decay can also occur much earlier. The origin of this effect lies in the temporal electrostatic stabilization of dipole in the charge-transfer state in DNA.
\end{abstract}

\section{Introduction}

Deoxyribonucleic acid (DNA) carries the genetic information of all cellular forms of life. DNA is usually found as a double helix, in which the nucleoside bases of the single strands are stacked upon each other, forming strong hydrogen bonds with the bases in the complementary strand (Watson-Crick configuration $\left.^{1}\right)$. Due to the absorbance of the bases in the harmful ultraviolet (UV) region of the spectrum (wavelength $<400 \mathrm{~nm}$ ), DNA is vulnerable to photochemical damage. To protect the genetic information, highly elaborate mechanisms have evolved to repair damaged DNA. ${ }^{2-4}$ More important, however, is the robustness of DNA with respect to UV damage. Indeed, when arranged in the Watson-Crick configuration, the isolated base pairs have an extremely short excited-state lifetime, suggesting a high photostability. Excited-state decay measurements of bases and model base pairs suggest a subpicosecond repopulation of the ground state. ${ }^{5,6}$ Recent experiments by Abo-Riziq and coworkers indicate that the excited state of an isolated cytosineguanine $(\mathrm{C}-\mathrm{G})$ base pair has a lifetime in the order of a few

\footnotetext{
Max-Planck-Institute for Biophysical Chemistry.

$\div$ Imperial College.

(1) Watson, J. D.; Crick, F. H. C. Nature 1953, 171, 737-738

(2) Crespo-Hernandez, C. E.; Cohen, B.; Hare, P. M.; Kohler, B. Chem. Rev. 2004, 104, 1977-2019.

(3) Kraemer, K. H. Proc. Natl. Acad. Sci. U.S.A. 1997, 94, 11-14.

(4) Sinha, R. P.; Hader, D. P. Photochem. Photobiol. Sci. 2002, 1, 225-236.

(5) Schultz, T.; Samoylova, E.; Radloff, W.; Hertel, I. V.; Sobolewski, A. L.; Domcke, W. Science 2004, 306, 1765-1768.

(6) Peon, J.; Zewail, A. H. Chem. Phys. Lett. 2001, 348, 255-262.
}

tens of femtoseconds. ${ }^{7}$ Quantum chemistry calculations, including dynamic electron correlation, by Domcke and co-workers suggest that this ultrafast deactivation may be triggered by a barrierless single proton transfer in the excited state. ${ }^{8,9}$

Here we present ab initio and mixed quantum/classical (QM/ $\mathrm{MM}$ ) molecular dynamics (MD) simulations ${ }^{10}$ of a photoexcited $\mathrm{C}-\mathrm{G}$ base pair in gas phase and embedded in DNA (Figure 1). The results of the simulations provide detailed structural and dynamical insights into the ultrafast radiationless deactivation mechanism at a resolution well beyond that of current experiments. According to the simulations, photon absorption to the charge-transfer state $(\mathrm{CT}$, Scheme $1 \mathrm{a} \rightarrow \mathrm{d})$ promotes transfer of a proton from the guanine to the cytosine (Figures 2 and 3 ). Although in principle not actually essential for the decay, the proton transfer indirectly enhances ultrafast radiationless deactivation of the excited state via an extended conical intersection (CI) seam. ${ }^{11}$

The CI has an unusual topology, in that there is only one degeneracy-lifting coordinate. This topology is the central mechanistic feature for the decay in both in vacuo and DNA.

(7) Abo-Riziq, A.; Grace, L.; Nir, E.; Kabelac, M.; Hobza, P.; de Vries, M. S. Proc. Natl. Acad. Sci. U.S.A. 2005, 102, 20-23.

(8) Sobolewski, A. L.; Domcke, W. Phys. Chem. Chem. Phys. 2004, 6, $2763-$ 2771.

(9) Sobolewski, A. L.; Domcke, W.; Hättig, C. Proc. Natl. Ac. Sci. U.S.A 2005, 102, 17903-17906.

(10) Warshel, A.; Levitt, M. J. Mol. Biol. 1976, 109, 227-249.

(11) Robb, M. A.; Garavelli, M.; Olivucci, M.; Bernardi, F. In Reviews in Computational Chemistry; Lipkowitz, K. B., Boyd, D. B., Eds.; WileyVCH: New York, 2000; Vol. 15, p 87. 


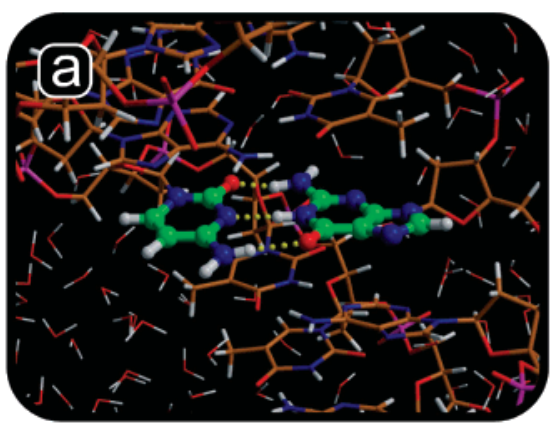

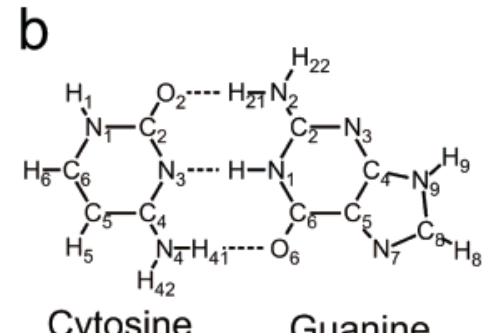

Figure 1. Cytosine-guanine base pair in the Watson-Crick configuration. (a) Snapshot taken from one of the QM/MM MD simulations of a 22 base pair DNA. The QM subsystem is shown in ball-and-stick representation; MM atoms are shown as sticks. Color code: carbon skeleton of the QM subsystem in green, MM carbon atoms in brown, nitrogen atoms in blue, phosphor atoms in magenta, oxygen atoms in red, and hydrogen atoms in white. (b) Schematic representation of the base pair in vacuo, defining the atom names used in the text. The image in (a) was created with Molscript ${ }^{30}$ and Raster3D. $^{31}$

Scheme 1. Valence Bond Representations of the Base Pair in the Relevant Electronic States

(d)<smiles></smiles>

CT

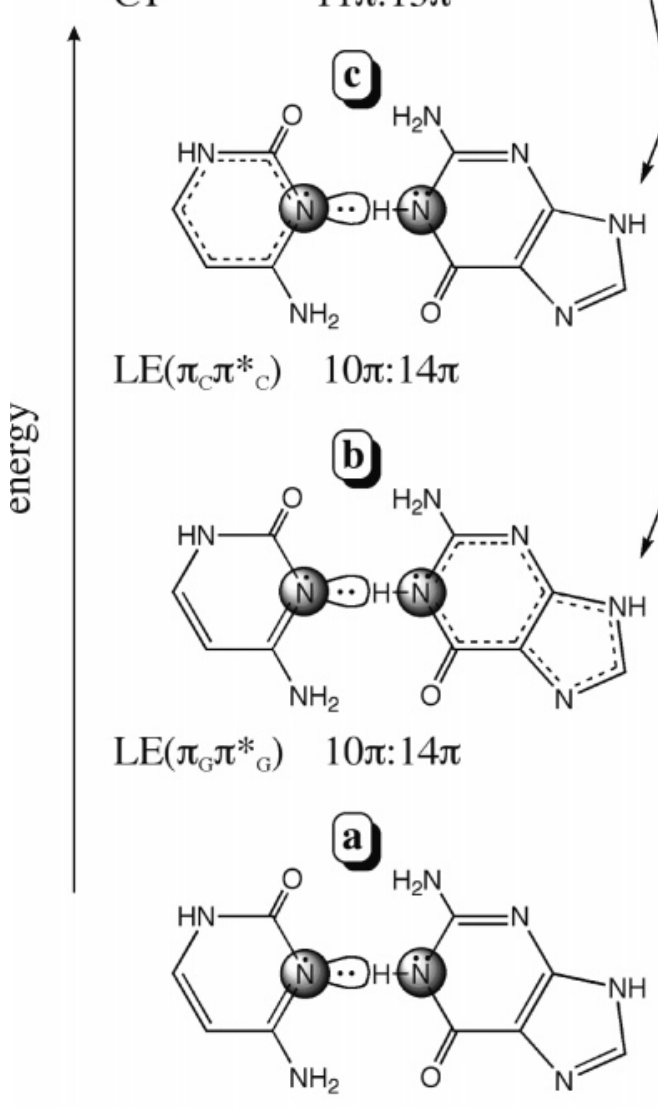

$\mathrm{S}_{0}$

$10 \pi: 14 \pi$ c'

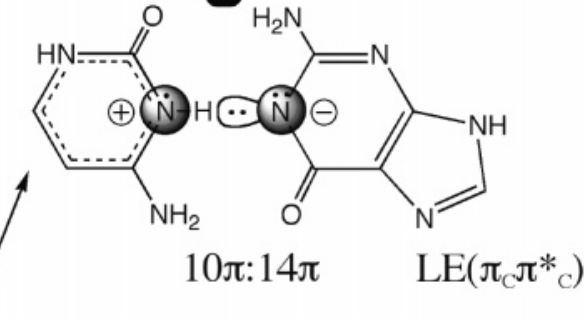
$\mathrm{H}_{2} \mathrm{~N}$

(b)<smiles>Nc1nc2[nH]cnc2c(=O)c2ccccc12</smiles>

$10 \pi: 14 \pi \quad \operatorname{LE}\left(\pi_{\mathrm{G}} \pi_{\mathrm{G}}{ }_{\mathrm{G}}\right)$

d'

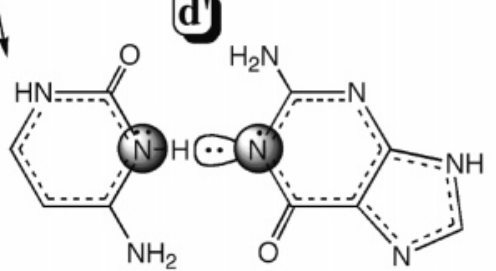

$11 \pi: 13 \pi$

CT

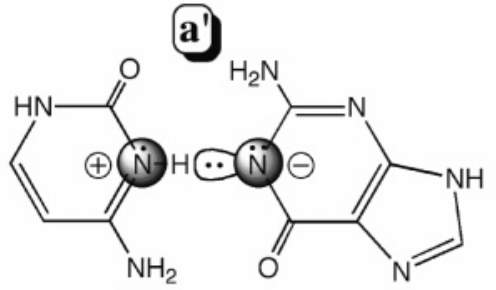

$10 \pi: 14 \pi$
$\mathrm{S}_{0}$

\section{Proton transfer}

Radiationless decay occurs along an extended hyperline that lies nearly parallel to the proton-transfer coordinate in the vicinity of the CT state minimum (Figure 2), indicating that the proton transfer itself is not directly responsible for the deactivation. The seam is displaced from the minimum energy proton-transfer path along a skeletal deformation of the bases. Decay can thus occur anywhere along the single proton-transfer coordinate, accounting for the remarkably short lifetime of the 


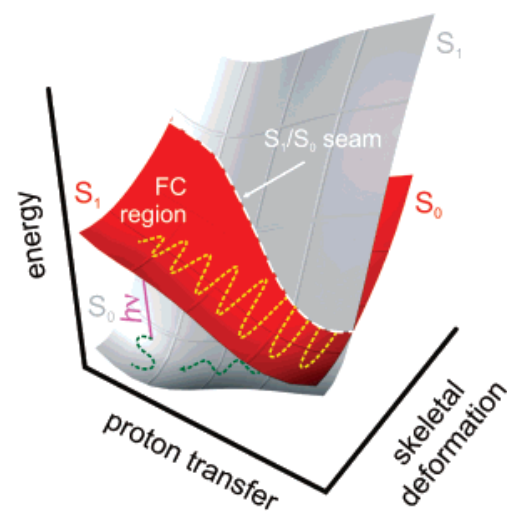

Figure 2. Potential energy surfaces of the excited and ground states of the cytosine-guanine base pair versus proton transfer $\left(\mathrm{N}_{1}-\mathrm{H}-\mathrm{N}_{3}\right)$ and skeletal deformation of the bonds (see Figure 5). The dashed yellow and green lines represent the path sampled in a typical trajectory. Motion along the protontransfer coordinate connects the Franck-Condon geometry to the minimum of the CT state. Nonradiative decay occurs along the intersection seam between the surfaces (conical intersection hyperline ${ }^{11}$ ). Note that the trajectory encounters the seam more than once and recrosses between the excited and ground states.

excited base pair. In vacuo, decay occurs after a complete proton transfer, whereas in DNA, decay can also occur much earlier. The origin of this effect lies in the temporal electrostatic stabilization of the dipole in the CT state in DNA.

\section{Methods}

To model the dynamics of the photoactivated $\mathrm{C}-\mathrm{G}$ base pair correctly in our MD simulations, energies and gradients of both the excited CT $\left(S_{1}\right)$ and ground states $\left(S_{0}\right)$ were calculated on-the-fly at the CASSCF/3-21G level of theory ${ }^{12}$ with a reduced active space of eight electrons in eight orbitals. The reaction starts on CT but ends on $S_{0}$. The "hops" between the two electronic states were modeled by surface selection at the CI seam. ${ }^{13}$ For all MD simulations, Gromacs $3.3^{14}$ with an interface ${ }^{13}$ to Gaussian $03^{15}$ was used.

The rather small active space of eight electrons in eight orbitals was validated using geometry optimizations of relevant excited-state minima and minimum energy crossing points for the isolated base pair. The full CASSCF active space for the $\mathrm{C}-\mathrm{G}$ base pair would require $24 \pi$ electrons distributed in $19 \pi$ orbitals. Neglecting the lowest energy nodeless and strongly occupied orbitals on each base and the corresponding virtual orbitals yields a manageable 20 electron, 15 orbital active space in which a single energy point calculation is feasible. ${ }^{16}$ The final eight electron-, eight orbital-reduced active space was selected from a calculation using the larger active space to enable the simultaneous description of the electronic ground state and the lowest excited charge-transfer state (see Supporting Information). The $\sigma$ orbitals involved in the transfer of the central proton were not included in the active space because the proton transfer involves heterolytic bond cleavage.

At the Franck-Condon geometry, the CT state appears, in our calculations, as the lowest excited state $\left(S_{1}\right)$ because of our particular selection of active space and orbitals, which biases this state relative to the locally excited (LE) states (see Supporting Information). Highly correlated computations by Domcke and co-workers ${ }^{8,9}$ show two LE

(12) Roos, B. O.; Taylor, P. R.; Siegbahn, P. E. M. Chem. Phys. 1980, 48, $157-171$.

(13) Groenhof, G.; Bouxin-Cademartory, M.; Hess, B.; de Visser, S. P.; Berendsen, H. J. C.; Olivucci, M.; Mark, A. E.; Robb, M. A. J. Am. Chem. Soc. 2004, 126, 4228-4232.

(14) van der Spoel, D.; Lindahl, E.; Hess, B.; Groenhof, G.; Mark, A. E.; Berendsen, H. J. C. J. Comput. Chem. 2005, 26, 1701-1718.

(15) Frisch, M. J.; et al. Gaussian 03, revision C.02; Gaussian, Inc.: Wallingford, CT, 2005

(16) Werner, H.-J.; et al. MOLPRO, version 2002.6; Birmingham, U.K., 2003. states in between the ground state and the charge-transfer state (Scheme $1 \mathrm{~b}, \mathrm{c})$. The relative energies of these states are within $40 \mathrm{~kJ} / \mathrm{mol}$ of the CT state. These LE states would be initially populated upon excitation because of their much larger oscillator strengths. ${ }^{8}$ However, as Domcke's results show, after vibrational relaxation, the LE states become rapidly higher in energy than the CT state (see Figure 6 in ref 8 ). Thus, as we will discuss in the next section, the active space used in our MD simulations is a good representation of the CT state, and there is good agreement (barrierless proton-transfer pathway, CT minimum structure, and surface crossing with the ground state) with highly correlated CASPT2 calculations by Domcke and co-workers. Moreover, as Domcke and co-workers have also shown, there is virtually no barrier to reach either an avoided or a surface crossing, which justifies initiating our molecular dynamics simulations directly in the CT state. Thus, we have not studied the vibrational relaxation taking place in one of the LE states, but rather we have focused on the single proton-transfer channel only.

The CASSCF computations are the main computational cost. At each step in the MD simulation, the appropriate CASSCF configuration interaction eigenvector was selected to compute the gradient. A diabatic "hop" from $S_{1}$ to $S_{0}$ was allowed when the energy gap was below a given threshold, and the eigenvector indicated that a crossing had been passed. We now briefly indicate the nature of the algorithm used. ${ }^{13}$ We use the symbol $C_{i}^{K}$ to refer to the vector for state $K$ at MD step $i$ ( $K=1$ for the ground state and $K=2$ for the excited state). Initially, $C_{i}{ }^{2}$ is used to compute the gradient. When the inner product $C_{i}^{2} \cdot C_{i-1}{ }^{2}$ is sufficiently small and the other inner product $C_{i}{ }^{1} C_{i-1}{ }^{2}$ approaches 1 , then the trajectory has passed a point of an avoided or a real crossing. Provided the energy difference is less than a threshold, we assume that the step $(i-1 \rightarrow i)$ has passed a point of real crossing, and we select the eigenvector $C_{i}{ }^{1}$ rather than $C_{i}{ }^{2}$ to compute the gradient at step $i$. Energy is obviously conserved, as the trajectory never leaves the diabatic surface. Of course, such a procedure only allows surface hopping on the multidimensional hyper line of the conical intersection. This procedure could lead to an underestimation of the crossing probability. However, our experience has shown that, in larger polyatomic systems, the conical intersection line is almost impossible to avoid because of its high dimensionality and most surface hops are, in practice, essentially diabatic.

The starting coordinates for the QM/MM simulations of the fully hydrated 22 base pair B-DNA molecule were extracted from the crystal structure of the human DNA/topoisomerase I complex, in which the 5-iodo-2'-deoxyuracils were replaced by thymines and a break in the strand was fixed (entry $1 \mathrm{~A} 31$ of the $\mathrm{PDB}^{17}$ ). The cytosine8-guanine37 base pair in the center of the molecule was described at the QM level. The remainder of the system was modeled with the Amber99 force field. ${ }^{18}$ The chemical bonds between the bases and the deoxyribose sugar rings connecting the QM and the MM subsystems were replaced by constraints, ${ }^{19}$ and the QM part was capped with two hydrogen link atoms. The forces on the link atoms were distributed over the two atoms of the original bonds using the lever rule. The QM system experienced the Coulombic field of all $\mathrm{MM}$ atoms within a sphere of $1.6 \mathrm{~nm}$; Lennard-Jones interactions between MM and QM atoms were added. To avoid overpolarization of the QM subsystem, the charges on the $\mathrm{MM}$ atoms at the QM/MM boundary were set to zero. The charges on the neighboring $\mathrm{MM}$ atoms were scaled to maintain an integer charge in the MM subsystem. In the QM/MM simulations, a small time step of $0.5 \mathrm{fs}$ was used, because no bond-length constraints were used in the QM subsystem.

To equilibrate the DNA and the solvent prior to the QM/MM simulations, the system was simulated classically for $1000 \mathrm{ps}$. The

(17) Redinbo, M. R.; Stewart, L.; Kuhn, P.; Champoux, J. J.; Hol, W. G. Science 1998, 279, 1504-1513.

(18) Wang, J. M.; Cieplak, P.; Kollman, P. A. J. Comput. Chem. 2000, 21, $1049-1074$.

(19) Hess, B.; Bekker, H.; Berendsen, H. J. C.; Fraaije, J. G. E. M. J. Comput. Chem. 1997, 18, 1463-1472. 


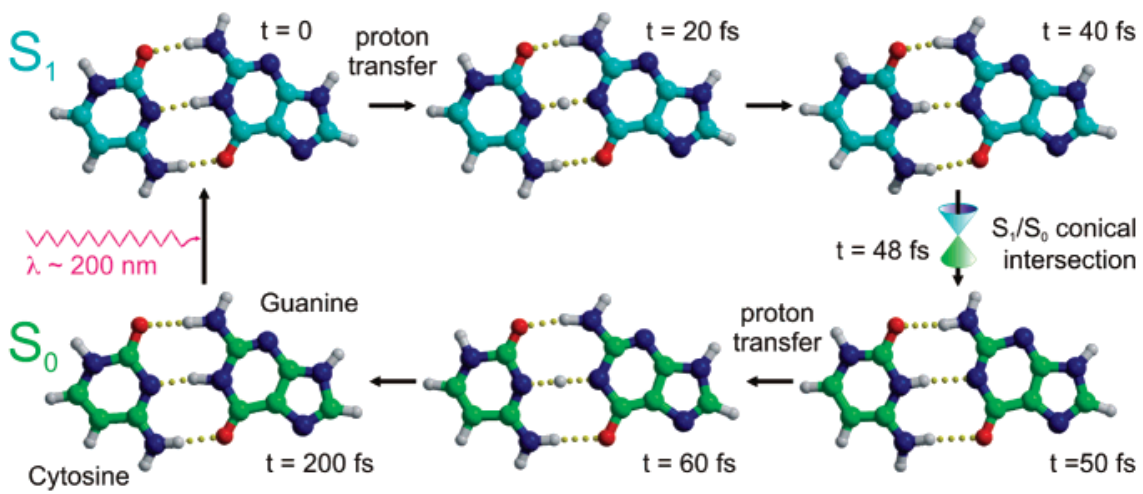

Figure 3. Photochemistry of the cytosine-guanine base pair in vacuum. Upon excitation to the charge-transfer state ( $S_{1}$, cyan carbon atoms) an electron transfer takes place from guanine to cytosine that is followed by a proton translocation (snapshot after $20 \mathrm{fs}$ ). The proton transfer leads to fast deactivation to $S_{0}$ (green carbons) via the conical intersection seam ( $48 \mathrm{fs}$ ). After returning to $S_{0}$, the proton is rapidly transferred back to the guanine base. The images are snapshots from one of the trajectories (run $\mathrm{r}$, Table 1).

simulation was performed in a rectangular $195 \mathrm{~nm}^{3}$ periodic box. After adding 6045 TIP4P water molecules, ${ }^{20}$ we inserted $42 \mathrm{Na}^{+}$ions to compensate for the net negative charge of the DNA. These ions were introduced by replacing the water molecules with the highest electrostatic potential. This was done in an iterative fashion, that is, after each water molecule was replaced with an ion, the electrostatic potential was recalculated. The final system contained 25627 atoms. Prior to the classical simulation, the system was energy-minimized for 200 steps using a steepest-descents algorithm. Subsequently, a 250-ps MM simulation was performed with harmonic position restraints on all DNA heavy atoms (force constant $10^{3} \mathrm{~kJ} \mathrm{~mol}^{-1} \mathrm{~nm}^{-2}$ ) for an initial equilibration of the water molecules and the ions. The MM simulations were run at constant temperature and pressure by coupling to an external bath $^{21}\left(\tau_{\mathrm{T}}=0.1 \mathrm{ps}\right.$ and $\left.\tau_{\mathrm{p}}=1.0 \mathrm{ps}\right)$. The LINCS algorithm ${ }^{19}$ was used to constrain bond lengths, allowing a larger time step of $2 \mathrm{fs}$ in the classical simulations. SETTLE ${ }^{22}$ was applied to constrain the internal degrees of freedom of the water molecules. A twin-range cutoff method was used for nonbonded interactions. Lennard-Jones interactions within $1.6 \mathrm{~nm}$ were calculated at every time step, as were the Coulombic interactions within $1.0 \mathrm{~nm}$. The particle mesh Ewald method was used to calculate long-range Coulombic interactions. ${ }^{23}$

To generate the initial conditions for the excited-state simulations, the systems were simulated for an additional $2 \mathrm{ps}$ in the ground state at the $\operatorname{CASSCF}(8,8)$ level. From this ground-state trajectory, 20 frames at equal time intervals were used as starting structures for the excitedstate MD simulations. The importance of generating multiple excitedstate trajectories has also been highlighted by Warshel and Chu. ${ }^{24}$

\section{Results and Discussion}

Figure 2 shows a "cartoon" of the potential energy surfaces of the cytosine-guanine base pair for the ground (gray) and excited states (red) along with the path of a typical MD trajectory (dashed yellow and green lines). The multidimensional surfaces are projected onto the proton-transfer coordinate and the single degeneracy-lifting coordinate. The first coordinate involves transfer of the central proton $\left(\mathrm{N}_{1}-\mathrm{H}-\mathrm{N}_{3}\right.$, Figure $\left.1 \mathrm{~b}\right)$, while the latter describes a skeletal deformation of the base pair. The position of the $S_{1} / S_{0}$ intersection seam between the ground and excited states controls the passage of the trajectory from the excited state to the ground state.

(20) Jorgensen, W. L. J. Am. Chem. Soc. 1981, 103, 335-340.

(21) Berendsen, H. J. C.; Postma, J. P. M.; van Gunsteren, W. F.; DiNola, A.; Haak, J. R. J. Chem. Phys. 1984, 81, 3684-3690.

(22) Miyamoto, S.; Kollman, P. A. J. Comput. Chem. 1992, 13, 952-962.

(23) Darden, T.; York, D.; Pedersen, L. J. Chem. Phys. 1993, 98, 10089-10092.

(24) Warshel, A.; Chu, Z. T. J. Phys. Chem. B 2001, 105, 9857-9871.
Table 1. Excited-State Lifetimes and Number of Surface Hops in 20 Simulations of an Isolated Cytosine-Guanine Base Pair in Vacuo $^{a}$

\begin{tabular}{ccccc}
\hline run & $\begin{array}{c}S_{1} \text { lifetime (fs) } \\
\text { (first hop) }\end{array}$ & $\begin{array}{c}S_{1} \text { lifetime (fs) } \\
\text { (last hop) }\end{array}$ & $\begin{array}{c}\text { No. of } S_{1} / S_{0} \\
\text { recrossings }\end{array}$ & $\begin{array}{c}N_{3}-\text { H distance } \\
\text { last hop }(\AA)\end{array}$ \\
\hline $\mathrm{a}$ & 19 & 56 & 2 & 1.09 \\
$\mathrm{~b}$ & 49 & 223 & 9 & 0.92 \\
$\mathrm{c}$ & 27 & 29 & 1 & 1.19 \\
$\mathrm{~d}$ & 51 & 188 & 6 & 0.95 \\
$\mathrm{e}$ & 28 & 70 & 2 & 0.98 \\
$\mathrm{f}$ & 28 & 49 & 1 & 1.12 \\
$\mathrm{~g}$ & 30 & 207 & 8 & 1.04 \\
$\mathrm{~h}$ & 48 & 118 & 3 & 0.88 \\
$\mathrm{i}$ & 37 & 163 & 6 & 1.09 \\
$\mathrm{j}$ & 28 & 57 & 2 & 1.22 \\
$\mathrm{k}$ & 25 & 31 & 1 & 1.23 \\
$\mathrm{l}$ & 45 & 69 & 2 & 1.10 \\
$\mathrm{~m}$ & 46 & 166 & 4 & 1.04 \\
$\mathrm{n}$ & 35 & 64 & 3 & 1.09 \\
$\mathrm{o}$ & 28 & 50 & 2 & 0.98 \\
$\mathrm{p}$ & 31 & 163 & 5 & 0.98 \\
$\mathrm{q}$ & 30 & 106 & 4 & 0.99 \\
$\mathrm{r}$ & 48 & 48 & 0 & 1.08 \\
$\mathrm{~s}$ & 49 & 128 & 2 & 0.95 \\
$\mathrm{t}$ & 46 & 81 & 2 & 0.96 \\
\hline
\end{tabular}

${ }^{a}$ The two lifetimes reported in the second and third column refer to the time until the first and last surface hops to the ground state, respectively.

Dynamics of the Isolated Cytosine-Guanine Base Pair. In the gas phase, 20 excited-state simulations were initiated, each from a different frame of the 2.0-ps ground-state trajectory. In these simulations, an excited-state lifetime between 19 and 223 fs was observed (Table 1). Most trajectories showed several recrossings between the $S_{1}$ and $S_{0}$ surfaces (Table 1, fourth column). Such recrossing phenomenon has also been observed experimentally for the diatomic sodium iodide gas. ${ }^{25}$ On average, three such recrossings were observed (Table 1). Simple exponential fits yielded decay times of $\tau=29 \pm 3$ fs and $\tau=89 \pm$ 4 fs until the first and final surface hop to $S_{0}$, respectively. These timescales can be compared to recent measurements that show a fast decay time on the order of a few tens of femtoseconds. ${ }^{7}$ Statistically, the number of trajectories may seem small but nevertheless yields a consistent mechanistic picture.

The excitation to the CT state, in which a single electron has been transferred from guanine to cytosine $(\mathrm{a} \rightarrow \mathrm{d}$, Scheme 1$)$,

(25) Rose, T. S.; Rosker, M. J.; Zewail, A. H. J. Chem. Phys. 1988, 10, 66726673 


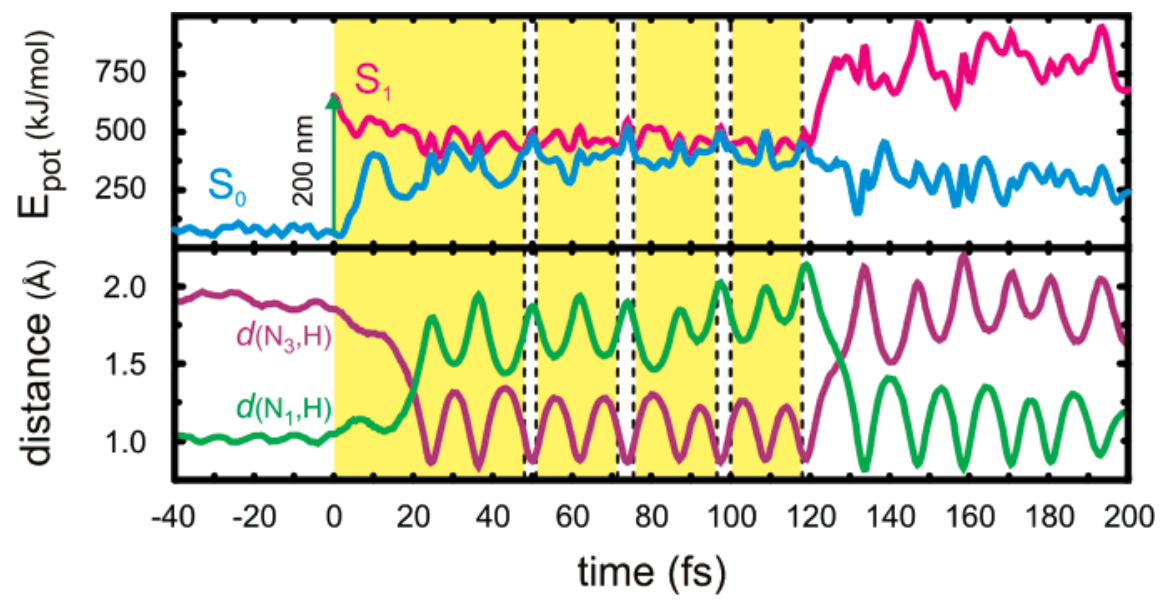

Figure 4. $S_{0}$ and $S_{1}$ potential energies during a simulation (top panel, run h in Table 1); distances of the central proton to the donor (guanine $\mathrm{N}_{1}$, Figure 1) and acceptor (cytosine $\mathrm{N}_{3}$ ) (bottom). Hops are indicated by the dashed lines; time intervals on $S_{1}$ are colored yellow.

Table 2. Bond Distances in Angstroms of the Cytosine (Left) and Guanine (Right) Bases (Figure 1b) at the Optimized Minima of the Charge Transfer (CT) State and of the Conical Intersection (CI) Seam

\begin{tabular}{|c|c|c|c|c|c|}
\hline \multicolumn{3}{|c|}{ cytosine } & \multicolumn{3}{|c|}{ guanine } \\
\hline bond & CT minimum & $\mathrm{Cl}$ minimum & bond & CT minimum & $\mathrm{Cl}$ minimum \\
\hline $\mathrm{N}_{1}-\mathrm{C}_{2}$ & 1.358 & 1.356 & $\mathrm{~N}_{1}-\mathrm{C}_{2}$ & 1.307 & 1.307 \\
\hline $\mathrm{C}_{2}-\mathrm{O}_{2}$ & 1.238 & 1.238 & $\mathrm{C}_{2}-\mathrm{N}_{2}$ & 1.317 & 1.317 \\
\hline $\mathrm{C}_{2}-\mathrm{N}_{3}$ & 1.353 & 1.354 & $\mathrm{C}_{2}-\mathrm{N}_{3}$ & 1.414 & 1.416 \\
\hline $\mathrm{N}_{3}-\mathrm{H}$ & 1.011 & 1.010 & $\mathrm{~N}_{3}-\mathrm{C}_{4}$ & 1.274 & 1.273 \\
\hline $\mathrm{N}_{3}-\mathrm{C}_{4}$ & 1.412 & 1.415 & $\mathrm{C}_{4}-\mathrm{C}_{5}$ & 1.442 & 1.441 \\
\hline $\mathrm{C}_{4}-\mathrm{N}_{4}$ & 1.373 & 1.377 & $\mathrm{C}_{5}-\mathrm{C}_{6}$ & 1.439 & 1.441 \\
\hline $\mathrm{C}_{4}-\mathrm{C}_{5}$ & 1.368 & 1.365 & $\mathrm{C}_{6}-\mathrm{O}_{6}$ & 1.247 & 1.246 \\
\hline $\mathrm{C}_{5}-\mathrm{C}_{6}$ & 1.402 & 1.405 & $\mathrm{C}_{6}-\mathrm{N}_{1}$ & 1.381 & 1.383 \\
\hline \multirow[t]{4}{*}{$\mathrm{C}_{6}-\mathrm{N}_{1}$} & 1.419 & 1.419 & $\mathrm{C}_{5}-\mathrm{N}_{7}$ & 1.368 & 1.366 \\
\hline & & & $\mathrm{N}_{7}-\mathrm{C}_{8}$ & 1.325 & 1.326 \\
\hline & & & $\mathrm{C}_{8}-\mathrm{N}_{9}$ & 1.398 & 1.397 \\
\hline & & & $\mathrm{C}_{4}-\mathrm{N}_{9}$ & 1.367 & 1.368 \\
\hline
\end{tabular}

induces a spontaneous transfer of the central proton from guanine to cytosine within a few femtoseconds (Figure 3 and Scheme $\left.1, d \rightarrow d^{\prime}\right)$. The bottom panel of Figure 4 shows the distance of this proton to its donor (guanine $\mathrm{N}_{1}$ ) and acceptor (cytosine $\mathrm{N}_{3}$ ) during a typical simulation. The last column of Table 1 lists the $\mathrm{N}_{3}-\mathrm{H}$ distance at the time of the last hop, which ranges from 0.88 to $1.23 \AA$. In the CI minimum, the $\mathrm{N}_{3}-\mathrm{H}$ distance is $1.01 \AA$ (Table 2), and thus the hopping geometries are all close to that CI minimum.

After the proton transfer, the system hits the conical intersection seam (Figure 4) and returns to the ground state, as indicated by the first dashed line. However, within a few femtoseconds, the seam is encountered again, and a second hop takes the system back to $S_{1}$ (Figure 4 ), where it stays until another hop occurs. These recrossings are a manifestation of a specific CI surface topology that we will discuss later. Because of this topology, diabatic trapping is possible, ${ }^{26}$ and in principle the system could remain locked, switching back and forth between the $S_{1}$ and $S_{0}$ states and never reaching the $S_{0}$ minimum. However, this was not observed in any of our simulations, and after a finite number of such recrossings, all trajectories eventually ended up on the ground-state surface. On average, the system spends $60 \mathrm{fs}$ in this diabatic trap.

The hops to $S_{0}$ are accompanied by a shift of negative charge from the cytosine back to the guanine $\left(d^{\prime} \rightarrow a^{\prime}\right.$, Scheme 1).

(26) Blancafort, L.; Hunt, P.; Robb, M. A. J. Am. Chem. Soc. 2005, 127, 33913399.
This charge transfer provides the driving force for the central proton $\left(\mathrm{N}_{1}-\mathrm{H}\right)$ to return to the guanine after the final hop, which happens within a few femtoseconds (Figures 2-4, Scheme 1, $\left.a^{\prime} \rightarrow a\right)$.

In five out of 20 simulations, the original Watson-Crick configuration was not restored, and the base pair eventually adopted a different tautomeric state, as shown in Figure 5. In three of these cases, the central proton remained bound to the cytosine $\left(\mathrm{N}_{3}-\mathrm{H}\right)$, and after the final surface hop to the ground state, a proton was transferred from the cytosine amino group $\left(\mathrm{NH}_{2}\right)$ to the guanine $\mathrm{O}_{6}$ oxygen atom instead. In the other two cases, the excess thermal energy released during the deactivation process induced a concerted double proton transfer in the ground state after the final radiationless transition, yielding the same tautomer. The system remained in this local minimum for the rest of the simulation, suggesting a barrier for retautomerization. This observation is in agreement with the experiments of Zewail and co-workers on model base pairs ${ }^{27}$ and was also seen in CPMD simulations. ${ }^{28}$ In solvated double-stranded DNA, where the bases are held tightly together by the phosphate sugar backbones, the excess thermal energy can be channeled away more efficiently, and such potentially mutagenic double protontransfer events were not observed (see below).

$S_{1} / S_{0}$ Conical Intersection Topology. Here we discuss the conical intersection that occurs between $\mathrm{a}^{\prime}$ and $\mathrm{d}^{\prime}$ (Scheme 1)

(27) Douhal, A.; Kim, S. K.; Zewail, A. H. Nature 1995, 378, 260-263.

(28) Markwick, P. R. L.; Doltsinis, N. L.; Schlitter, J. J. Chem. Phys. 2007, $126,45104-45111$. 


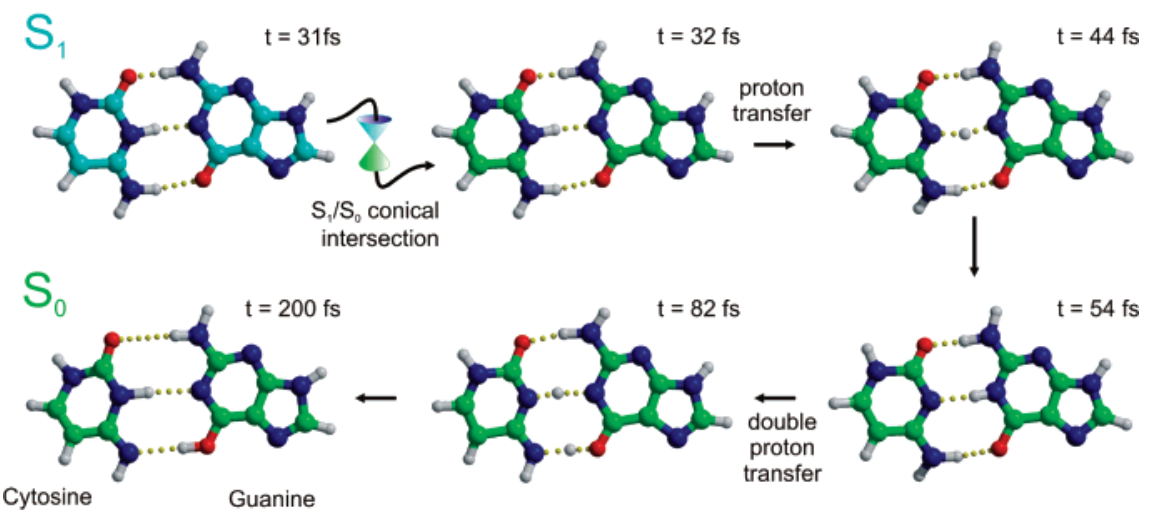

Figure 5. Formation of a different tautomer via concerted double proton transfer. After deactivation of the excited state $\left(S_{1}\right.$, cyan carbon atoms) via the conical intersection, the proton returns to the guanine base. The excess thermal energy that is released upon returning to $S_{0}$ (green carbon atoms) induces a spontaneous double proton transfer ( $82 \mathrm{fs})$, leading to the formation of a different tautomeric state ( $200 \mathrm{fs})$.

a

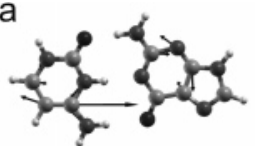

gradient difference vector

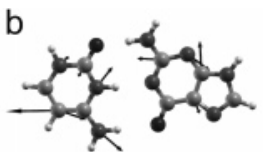

derivative coupling vector

Figure 6. Computed gradient difference (a) and derivative coupling vectors (b) at the optimized conical intersection geometry, which is almost identical to the $S_{1}$ minimum (Table 2). The plotted amplitudes are arbitrary. The vectors in (a) and (b) are obviously collinear and of opposite phase. Thus, they are linearly dependent and span a one-dimensional branching space.

in the region of the CT minimum (Figure 2). Photon absorption triggers a transition from the covalent ground state (Scheme 1a) with 10 and 14 electrons in the $\pi$ systems of the cytosine and guanine, respectively, to the zwitterionic CT state (Scheme 1d) with 11 and $13 \pi$ electrons, respectively. This CT state is diabatically correlated to the non-zwitterionic CT minimum with the central proton transferred to the cytosine (Scheme 1d').

We have optimized the excited-state CT minimum and an adjacent conical intersection. Table 2 lists the bond lengths at these critical points. The central proton is completely transferred to the cytosine in these configurations, with the $\mathrm{N}_{3}-\mathrm{H}$ bond distance of $1.011 \AA$ in the CT minimum and $1.010 \AA$ at the conical intersection (Table 2). This is in good agreement with Domcke's highly correlated calculations, ${ }^{8,9}$ which locate the CT minimum at $\mathrm{N}_{1}-\mathrm{H}$ distance of $2.0 \AA$, compared to $1.98 \AA$ in our calculations. In the isolated base pair, the CI minimum lies only $1 \mathrm{~kJ} / \mathrm{mol}$ above the CT minimum, thus indicating that the seam is encountered after adiabatic reaction on the CT state surface. We have not found other minima or other topological features between the Franck-Condon region and the CT minimum

At the conical intersection, the gradients of the ground and excited states are parallel (see Figure S1 in Supporting Information), indicating that the CI is sloped. The gradient difference vector and the interstate coupling vectors that span the branching space of the conical intersection are collinear and correspond to a skeletal deformation of the two bases (Figure 6). These vectors do not contain a first-order component of the proton transfer. The conical intersection therefore lies on a hyperline that is parallel to the proton-transfer coordinate (Figure 2). A quadratic analysis of the intersection seam revealed that the coupling to proton transfer is of second order and that the seam curves along this coordinate.

Because the derivative coupling and gradient difference vectors (which lift the $S_{1} / S_{0}$ degeneracy of the seam) are collinear, the branching space is one-dimensional. The conical intersection hyperline thus has a dimension of $(n-1)$ instead of $(n-2),{ }^{11}$ where $n$ is the number of degrees of freedom of the system. The unusual character of this $(n-1)$ dimensional sloped intersection seam means that it cannot be avoided, creating a diabatic trap for the CT state (see Figure S2 in Supporting Information where the seam has been mapped out as the point of degeneracy that lies nearest to the proton-transfer coordinate).

In summary, the $S_{1} / S_{0}$ intersection seam in the base pair is sloped and there is only one coordinate lifting the degeneracy. This topology accounts for the recrossings (partial diabatic trapping) that were observed in the dynamic simulations (Table 1, column 4). As schematically shown in Figure 2, vibrational motions along this coordinate repeatedly drive the system inand-out of the CI, until the base pair eventually returns to its stable ground-state minimum. The proton-transfer coordinate is perpendicular to the one-dimensional branching space and therefore does not lift the degeneracy. The seam is accessible anywhere along the proton-transfer coordinate, and in principle proton transfer is not essential for the $S_{1}$ decay. Rather, since in the excited CT state proton transfer is a barrierless downhill process, part of the excitation energy is rapidly channeled into excess vibrational energy of the other degrees of freedom (via intramolecular vibrational relaxation), including the skeletal deformation that leads to the intersection seam. Therefore, the proton transfer indirectly facilitates the ultrafast radiationless decay.

Dynamics of the Cytosine-Guanine Base Pair in DNA. In the previous subsection, we documented the mechanism that underlies the ultrafast excited-state decay process of an isolated base pair. To study how the DNA surroundings influence this process, we performed 20 excited-state QM/MM MD simulations of a cytosine-guanine base pair embedded in a fully hydrated double-stranded B-DNA molecule (Figure 1a). Also in DNA, the excited state rapidly decays via the CI seam. The average excited-state lifetimes of $\tau=48 \pm 4 \mathrm{fs}$ and $\tau=137 \pm$ 6 fs until the first and final surface hop to $S_{0}$, respectively (Table $3)$, are somewhat longer than in vacuo. However, these time scales are still very short, and thus both in DNA and in vacuo, the deactivation can be considered an ultrafast process. An average of four $S_{1} / S_{0}$ recrossings were observed in the DNA, 
Table 3. Excited-State Lifetimes and Number of Surface Hops in 20 Simulations of a Cytosine-Guanine Base Pair in Hydrated DNA $^{a}$

\begin{tabular}{lcccc}
\hline run & $\begin{array}{c}S_{1} \text { lifetime (fs) } \\
\text { (first hop) }\end{array}$ & $\begin{array}{c}S_{1} \text { lifetime }(\mathrm{fs}) \\
\text { (last hop) }\end{array}$ & $\begin{array}{c}\text { No. of } S_{1} / S_{0} \\
\text { recrossings }\end{array}$ & $\begin{array}{c}\mathrm{N}_{3} \text { H distance } \\
\text { last hop }(\AA)\end{array}$ \\
\hline $\mathrm{a}$ & 26 & 352 & 12 & 0.99 \\
$\mathrm{~b}$ & 27 & 87 & 3 & 0.94 \\
$\mathrm{c}$ & 28 & 102 & 5 & 1.35 \\
$\mathrm{~d}$ & 26 & 26 & 0 & 1.15 \\
$\mathrm{e}$ & 30 & 98 & 3 & 1.04 \\
$\mathrm{f}$ & 28 & 138 & 1 & 1.05 \\
$\mathrm{~g}$ & 58 & 172 & 10 & 1.09 \\
$\mathrm{~h}$ & 174 & 176 & 1 & 0.94 \\
$\mathrm{i}$ & 181 & 205 & 1 & 0.88 \\
$\mathrm{j}$ & 30 & 211 & 4 & 1.15 \\
$\mathrm{k}$ & 12 & 101 & 2 & 1.00 \\
$\mathrm{l}$ & 11 & 11 & 0 & 1.89 \\
$\mathrm{~m}$ & 23 & 197 & 7 & 1.04 \\
$\mathrm{n}$ & 27 & 128 & 6 & 0.90 \\
$\mathrm{o}$ & 25 & 68 & 1 & 1.07 \\
$\mathrm{p}$ & 67 & 498 & 13 & 1.08 \\
$\mathrm{q}$ & 122 & 259 & 6 & 1.19 \\
$\mathrm{r}$ & 30 & 84 & 2 & 1.00 \\
$\mathrm{~s}$ & 11 & 87 & 2 & 1.14 \\
$\mathrm{t}$ & 145 & 145 & 0 & 1.83 \\
\hline
\end{tabular}

${ }^{a}$ The two lifetimes reported in the second and third columns refer to the time until the first and last surface hops to the ground state, respectively.

one more than in vacuo. The $\mathrm{N}_{3}-\mathrm{H}$ distances at the final crossings (last column) ranged between 0.88 and $1.89 \AA$, spanning the full extent of the CI seam.

The $\mathrm{N}_{3}-\mathrm{H}$ distances at the last hop can be divided into two subpopulations. Most of the hops occur near the CT minimum, but in two of the 20 DNA trajectories the $S_{1} / S_{0}$ seam was hit before proton transfer could occur (runs 1 and t, Table 3). In these cases no recrossings were observed, which is a natural consequence of the extended seam (Figure 2). However, this part of the seam is not accessible in the gas phase. These observations suggest that the DNA environment can temporarily lower the energy of the charge-transfer state and thus bring the crossing seam closer to the Franck-Condon region than in vacuo. This conjecture was tested by computing the overall electrostatic stabilization of a CT dipole model created by placing a point charge in the center of each six-membered ring. The total electrostatic potential was then evaluated at every frame of the trajectories. In runs 1 and t, the CT dipole is indeed stabilized by the DNA environment by around $100 \mathrm{~kJ} / \mathrm{mol}$.

In DNA, the base pair always returns to the initial WatsonCrick configuration after deactivation, and no double protontransfer events were observed. The interactions with the rest of the DNA keep the base pairs tightly together. In this respect, the base pair is more photostable in DNA than in vacuo, where the recovery of the original ground-state Watson-Crick configuration was only observed in $75 \%$ of the cases. In the other $25 \%$ a wrong tautomer was formed (see above).

By including only a single base pair in the QM region, we have excluded the possibility of observing possible intrastrand excimer formation. Recent experiments by Kohler and coworkers on an (AT) $)_{18}$ B-DNA molecule revealed that excitedstate relaxation mainly involves such intrastrand excimer formation. ${ }^{29}$ The measured excited-state lifetime was about $50-$ $150 \mathrm{ps}$, indicating that the ultrafast proton transfer is not

(29) Crespo-Hernandez, C. E.; Cohen, B.; Kohler, B. Nature 2005, 436, 1141 1144.

(30) Kraulis, P. J. J. Appl. Crystallogr. 1991, 24, 946-950.

(31) Merritt, E. A.; Bacon, D. J. Methods Enzymol. 1997, 277, 505-524. the predominant decay channel in $(\mathrm{AT})_{18}$. This observation suggests that also for DNA containing $\mathrm{C}-\mathrm{G}$ base pairs such excimer formation could play an important role in the decay process. We must assume that there could be a competition between entering the charge-transfer state of the base pair and the excimer formation. The efficiency of the first process is strongly dependent on the hydrogen-bonding configuration, as Domcke and co-workers have shown. ${ }^{9}$ All our QM/MM simulations were initiated from ideal Watson-Crick configurations, with three hydrogen bonds between the bases. These configurations were by far more predominant during a 50-ns classical ground-state DNA simulation than partially unpaired base pairs. Entering the CT state from such partially unpaired configurations could be more difficult, leading to a different decay process. Studying alternative pathways such as excimer formation is a formidable task and is beyond the scope of the present article.

To summarize, the main features of radiationless decay in the $\mathrm{C}-\mathrm{G}$ base pair are a barrierless single proton transfer on the CT state and an extended conical intersection seam between the CT and ground state that is encountered in the vicinity of the CT minimum. Our computations are at the limit of what is feasible for "on-the-fly" QM/MM dynamics simulations. We do, however, recognize the limitations of our methods, but as our calculations have been calibrated against more accurate calculations, ${ }^{8,9}$ one can have a high degree of confidence at the qualitative level in the computed mechanism.

\section{Conclusions}

Understanding the dynamics of the ultrafast deactivation processes in the cytosine-guanine base pair represents a major challenge. Here we demonstrated that, by using an ab initio MD strategy with explicit surface hopping, it is possible not only to reproduce the experimental excited-state lifetime of the isolated cytosine-guanine base pair, but also to provide detailed mechanistic insights into the deactivation process. The important mechanistic aspect of the CI surface topology is the presence of an extended seam parallel to the reaction path, so that decay can occur in principle anywhere along the proton-transfer coordinate via a skeletal deformation. In the gas phase, the reaction path is sufficiently steep along the proton-transfer coordinate for the system to become partially equilibrated in the charge-transfer state minimum before radiationless decay. In the DNA, this process appears to be prevalent as well. However, decay was also observed occasionally near the Franck-Condon region, because of electrostatic stabilization of the CT resonance structure (Scheme 1d) by the DNA surroundings. We hope that this work will stimulate further experimental and theoretical studies to validate our conclusions, in particular, the ultrafast decay time and the predicted oscillatory repopulations of the excited state.

Acknowledgment. L.V.S. thanks the Boehringer Ingelheim Fonds for a Ph.D. scholarship. We thank Phineus Markwick for raising our interest in this subject. This work has been supported by EPSRC U.K. (Grant GR/S94704/01). Support from the EU Nanomot project (Grant 29084) is thankfully acknowledged. We thank the EPSRC National Service for Computational Chemistry Software (www.nsccs.ac.uk) for enabling us to run the MOLPRO calculations. Finally, we thank Ira Tremmel for carefully reading the manuscript and for valuable suggestions. 
Supporting Information Available: Cartesian coordinates and energies of optimized CASSCF structures; complete refs 15 and 16; discussion of the selection of active space orbitals; a picture of the $S_{1}$ and $S_{0}$ gradients at the CI minimum (Figure $S 1$ ); the computed cross section of the CI seam (Figure S2) along the single proton-transfer coordinate (PDF). Three animations of deactivation trajectories in vacuo and in the DNA, showing formation of the original Watson-Crick configuration in vacuo, formation of a different tautomer, and formation of the original Watson-Crick configuration in DNA (MPEG1). This material is available free of charge via the Internet at http://pubs.acs.org.

JA069176C 